\title{
Research on Multi-Sensor Fusion of Layered Intelligent System for Indoor Mobile Robot
}

\author{
Yingzhong Tian ${ }^{1,2}$, Xu Gao ${ }^{1}$, Mingxuan $\operatorname{Luan}^{1}$ and Long $\mathrm{Li}^{1,2, *}$ \\ ${ }^{1 *}$ School of Mechatronic Engineering and Automation, Shanghai University, Shanghai, 200072 CHINA \\ ${ }^{2}$ Shanghai Key Laboratory of Intelligent Manufacturing and Robotics, Shanghai 200072, China \\ ${ }^{*}$ Corresponding author
}

\begin{abstract}
-as the demand for smart home grows, robot manufacturers develop new indoor mobile robots, which allow for intelligent and high accuracy of the robot system. However, there is not such a standard system. In order to solve the above problems, this work focuses on the main problems of multilayer intelligent system on the indoor mobile robot: the recognition of voice information with semantic understanding and coordinate matching, which guiding the robot through the internal positioning and navigation algorithm to reach the matching coordinates. The proposed Multi-sensor fusion of layered intelligent system integrates different modules where each module encapsulates a number of related algorithms responsible for robot control in human-robot collaboration, such as the AMCL locating algorithm. The goal is to extract high-level voice information from human-robot conversions and control the robot to the target point. The system consists of several modules as sensor fusion, speech recognition, semantic understanding, coordinate matching, map construction, robot positioning, path planning and feedback processing. The general architecture and main approaches are presented as well as the future developments planned.
\end{abstract}

Keywords-multi-sensor fusion; layered intelligent system; indoor mobile robot; several modules

\section{INTRODUCTION}

With the development of robot technology, the robot has attracted people's attention in service industry especially since the introduction of Industry 4.0. Service robots are a key part of life and business applications today, including indoor handling, family escorts and shopping malls navigation. Different from the industrial robots, Service robot must have good man-robot interaction such as listening to people's words and precise arrive the target which people want to. To ensure that the above tasks intelligent, efficient and accurate completion, Robot need a reasonable system [1].

From the research results at home and abroad, the system the indoor mobile robot is no uniform. Minguez J (Minguez J, Montesamo L and Lamiraux F, 2006) proposals a hybrid realtime mobile robot platform [2]. Pomerleau F (Pomerleau F, Colas F, Siegwart R, 2013) puts forward a kinds of embedded system software platform named TOPPERS [3].da Silva, AR(da Silva, Machado, AMC, 2016) is focused on Control of Mobile Robots with Amorphic Architecture[4]. Wei Ren (Ding-Xin He, Jin Zhao, 2012) research on A Component Based Hybrid Embedded Framework for Mobile Robot [5]. A hybrid-processors real-time embedded Linux Platform which can be used in mobile robot is presented in [6].

This work focuses on the main problems of multilayer intelligent system on the indoor mobile robot. Perhaps this system will become a common system for mobile robots in the future.

\section{SYSTEM ARCHITECTURE}

\section{A. Overview}

The Multi-sensor fusion of layered intelligent system include three parts: Intelligent interaction, Algorithm processing and Real-time control .they are constituted of the following modules: sensor fusion, speech recognition, semantic understanding, coordinate matching, map construction, robot positioning, path planning and feedback processing, as shown in Figure 1. The most primary inputs for the system are voice message of the targets and the sensor feedback. Feature sets and $2 \mathrm{D}$ models of the targets are provided beforehand in the forms of 2D map and serve as a knowledge base for Positioning and path planning of the robot, as well as for the visualization. Sensor selection and parameter configuration are key elements of environmental construction, such as sensor selection for map construction, selection of obstacle avoidance sensors.

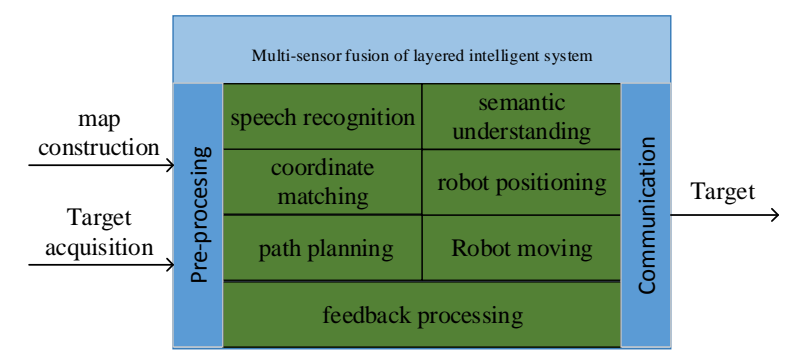

FIGURE I. MULTI - SENSOR FUSION OF LAYERED INTELLIGENT SYSTEM

This system includes intelligent interaction, algorithm processing and real-time control of three parts, these three parts will also be three systems of Android, Linux and ARM to complete, between them to communicate with Ethernet. Which under the Android system, the use of the letter fly voice development package, the interface can touch both control, Linux system using the Ros library to reduce the code repetition rate, ARM use keil development tools. It is the three 
parts by their most efficient system to complete, making the whole system more efficient, modular, and easy to secondary development, expansion and debugging.

To implement the proposed Multi-sensor fusion of layered intelligent system, in our laboratory an experimental platform has been established as shown in Figure 2 (A). The system includes a lot of sensors, controllers, and drives and so on as shown in Figure 2 (B).

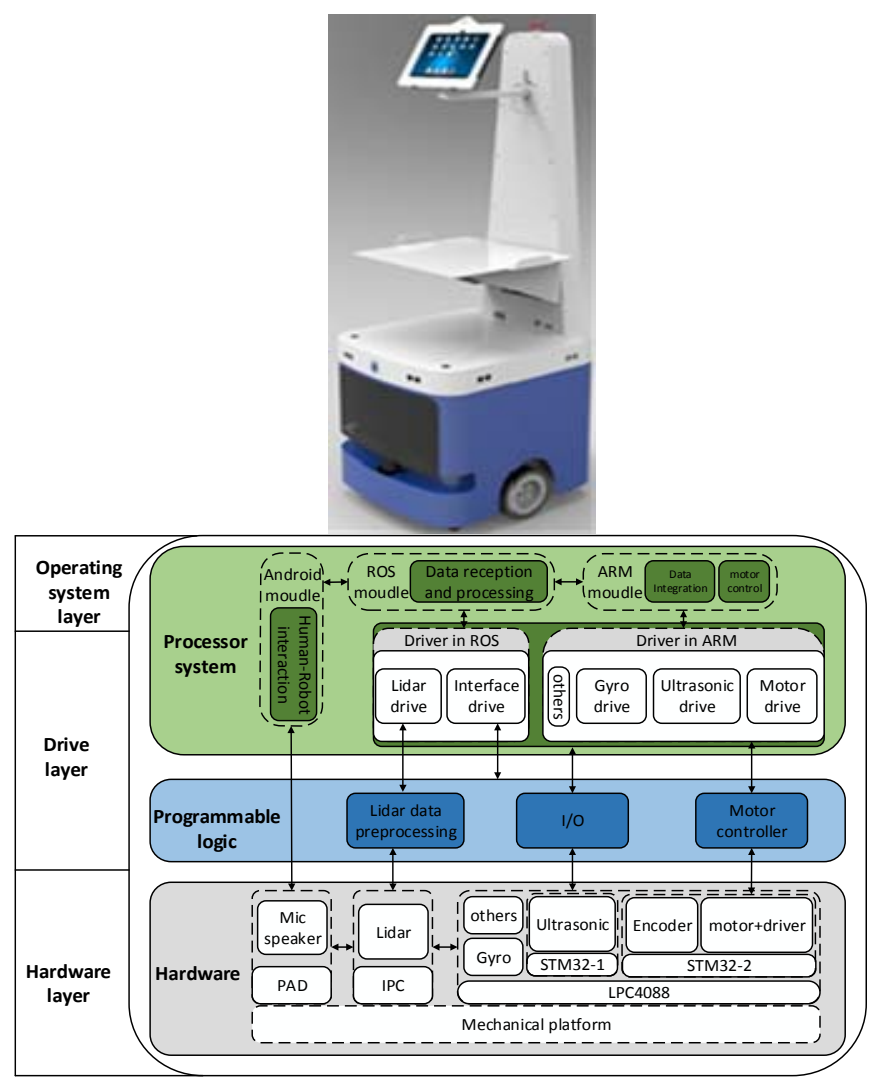

FIGURE II. THE EXPERIMENTAL PLATFORM: (A) EXPERIMENTAL PLATFORM, (B)FRAMEWORK

\section{B. Module Description and Workflow}

The main workflow our proposed Multi-sensor fusion of layered intelligent system is depicted in Figure3. Three black dashed boxes represent the three parts connected via Ethernet and the four red dotted wireframes represent the four major processes of the system. The workflow comprises four major processes which are noted as follows.

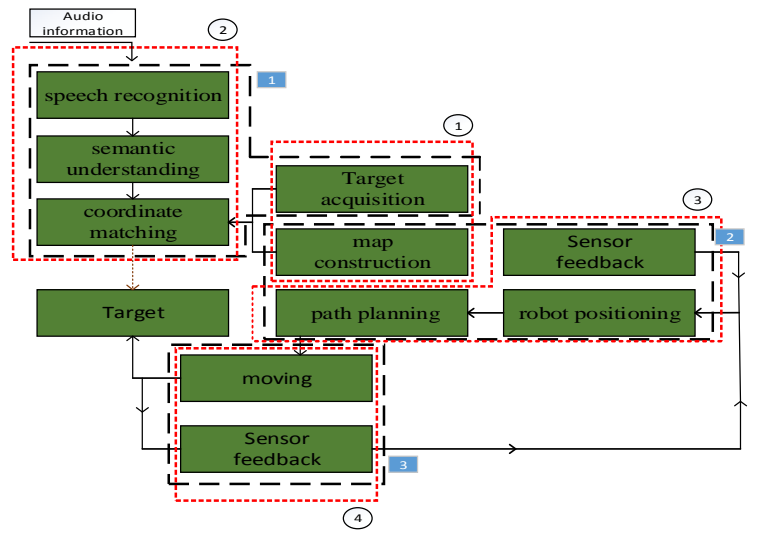

FIGURE III. THE WORKFLOW

1) This process consists of two parts: map building and coordinate acquisition. The map building makes the unknown environment into a known environment and it is also the visualization of robot movement. The Visualization module displays the calibrated image at the selected viewpoint or the image resulting from the fusion. In our experimental platform, a laser radar sensor produced by Cisco for map building. Target acquisition is matching the keywords extracted from the semantic understanding of human words with the absolute coordinates of the environment. The keywords and the absolute coordinates make up a unit and many of these units make up a matching database. For example: the keywords is kitchen and the absolute coordinates is (20 8030$)$.Both of them make up a unit looking like this kitchen (20 8030$) .20$ represents the $x$ coordinates, 80 represents the $\mathrm{x}$ - coordinates and 30 represents the rotation angle.

2) After receiving the voice information from the microphone, the robot will perform the following steps: the speech recognition, semantic understanding, and extract the keywords. According to the keyword, the interactive system will be get the target coordinates based on the matching database established in the previous step, and the data transmitted through Ethernet to next procedure

3) In this layer, a lot of motion algorithm are executed. Received the target coordinates from the transmission layer, this layer system will make a global path planning to the robot according to path planning algorithm. In the movement of the process, this layer system get information from many different sensors, and fuse those data in order to improve the performance of the robot such as updating the position of robot in real time. For the experimental platform mentioned in this article, when the robot encounter obstacles, the layer system can make a corresponding local path planning to achieve the purpose of obstacle avoidance based on fusing data from different sensor. Through the above different complex operations, this layered system will send specific data to next procedure

4) This process is the robot motion control layer. The motion control card receives data signal from previous procedure and sends the pulse signal to the driver driving the motor to rotate. The coordinates of robot will feedback to the upper layer through the sensors such as encoder. Control 
instructions and feedback information has been non-stop iterative transmission until the robot reach the target location, and the whole process to end.

\section{TOOLS AND METHODS}

As mentioned above, the proposed Multi-sensor fusion of layered intelligent system is developed on the basis of several software frameworks (ROS, keil) and voice processing libraries (Xunfei voice).

\section{A. Tools}

\section{1) ROS}

ROS (Robot Operating System) [7] is a software framework for robot software development. It provides standard operating system services such as hardware abstraction, low-level device control implementation of commonly-used functionality, message-passing between processes, and package management. ROS is composed of two main parts: the operating system ros as described above and ros-pkg, a suite of user contributed packages that implement functionality such as simultaneous localization and mapping, planning, perception, simulation etc[8].

The move_base provide path planning according to the reference message, so that the mobile robot to reach the specified location. Another package that plays a significant role for our purpose is $t$, which keeps track of multiple coordinate frames over time. tf maintains the relationship between coordinate frames in a tree structure buffered in time, and enables the transform of points, vectors, etc. between any two coordinate frames at any desired point in time

\section{2) Xunfei voice}

Xunfei Voice is an open intelligent interactive technology service platform and provides intelligent human-computer interaction solution. As a comprehensive intelligent humancomputer interaction platform, it provides the world's leading voice synthesis, speech recognition, semantic understanding and other technologies. Developers can choose the program according to their needs, so that intelligent human-computer interaction technology is more simple and practical.

\section{3) Keil}

Keil is a software framework for Microcontrollers (MCU) software development. It provides rich library functions, powerful integrated development and convenient debugging tools. It is the most common software development tools for various embedded processors today. $\mathrm{C}$ code is the programming language for Keil.

\section{B. Methods}

There have been two common systems in robotic control area [6].One is consisted of intelligent interaction and Realtime control, the disadvantage of this system is that it cannot carry out complex algorithm operations. Robot positioning navigation can only rely on a single sensor information, such as magnetic stripe navigation or two-dimensional code navigation. Another is consisted of intelligent interaction and Real-time control, the disadvantage of this system is that the interaction is relatively poor, the robot has no way to identify the human language, or the recognition rate is very low.

In order to solve the above problem, we propose the system model of this paper as shown in Figure 4and use multi-sensor fusion technology to improve the performance of the robot, such as improving positioning accuracy, optimizing the path and enhancing security. Next, for our laboratory robot platform as shown in Figure 4, we will describe one of our fusion technology in Robot positioning.

We used a total of three sensor are respectively gyro, encoder and Lidar in Robot positioning. The specific process as shown in Figure 5.

- $\quad$ First, get the robot's sports model by fusing the data of Encoder and gyroscope in Mileage calculation method, this step can also be called particle sampling according to positioning algorithm.

- $\quad$ Second, get the observation model through making use of the massage which produced in matching laser data with finished map to update the weight of the particle.

- $\quad$ Last, particle resampling in accordance with updated data in time and get precise position.

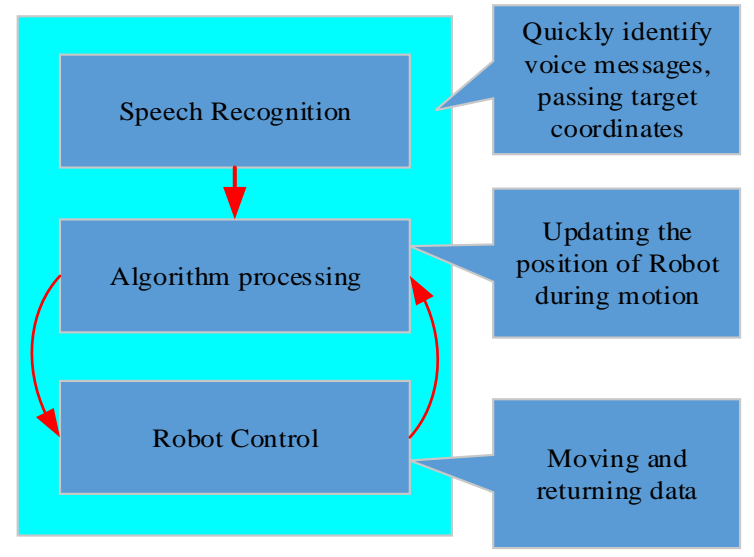

FIGURE IV. THE MULTI-SENSOR FUSION OF LAYERED INTELLIGENT SYSTEM CONTROL

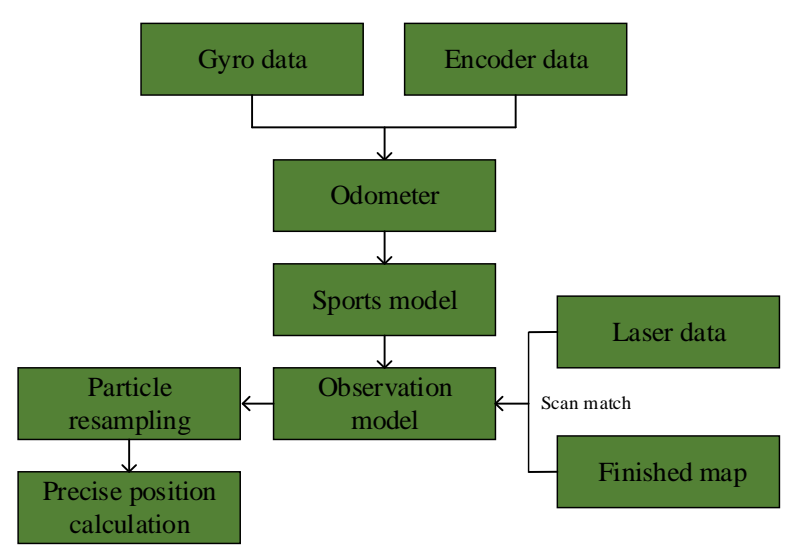

FIGURE V. THE PROCESSING OF ROBOT POSITIONING 


\section{CONCLUSION AND FUTURE}

\section{A. Conclusion}

In this paper, we introduce Multi - sensor fusion of layered intelligent system for indoor mobile robot. The system have three parts, each of which has its own best work area. Intelligent interaction (Xunfei voice), Algorithm processing (ROS) and Real-time control (Keil) deal with human-robot interaction, data processing and controlling the motor, respectively. As mentioned in this article, the three parts of the system is consist of many modules supported by several different sensors. The use of multi-sensor fusion technology to improve the performance of the robot

\section{B. Future}

To continue the development of our proposed Multi-sensor fusion of layered intelligent system, more efforts will be made on implementing the sensors processing modules in addition to the completed ones. Optimizations will be made on the system to achieve the two main goals:

- $\quad$ By changing the number and type of sensors, we can make the system available to other scenes of service robots.

- $\quad$ Develop multiple robots to work together using Multisensor fusion of layered intelligent system is also later research.

\section{ACKNOWLEDGMENT}

This research work supported by National Key Technology Support Program of China (2015BAF10B01), Science and Technology Commission of Shanghai Municipality (15111104002, 15111106302, 16111107802, 16111108202), Shanghai Training and Support Program for University Youth Teachers (ZZSD15050) and Innovation Program of Shanghai Municipal Education Commission (2015Z102800006)

\section{REFERENCES}

[1] A. Sangiovanni-Vincentelli and G. Martin, "Platform-based design and software design methodology for embedded systems," IEEE Design \& Test of Computers, vol. 18, pp. 23-33, Nov-Dec 2001.

[2] Minguez J, B. Montesano L, Lamiraux F. Metric-based iterative closest point scan matching for sensor displacement estimation[J]. IEEE Transactions on Robotics, 2006,22(5):1048-1054 .

[3] Pomerleau F, Colas F, Siegwart R, et al. Comparing ICP variants on real-word data sets: open source library and experimental protocol[J]. Autonomous Robotics, 2013,24:133-148

[4] da Silva, A. R.; Machado, A. M. C. Control of Mobile Robots with Amorphic Architecture.IEEE Latin america transactions,2016,14:16231635.

[5] Wei Ren, Ding-xin He, Jin Zhao , A Component Based Hybrid Embedded Framework. 2012, 13:16-19

[6] K. M. Goh, et al., "Hybrid-processors real-time embedded Linux platform," in TENCON 2009. 2009 IEEE Region 10 Conference, Piscataway, NJ, USA, 2009, pp. 1-5.

[7] ROS.org. [Online]. Available: http://wiki.ros.org/

[8] M. Quigley, et al., "ROS: an open-source Robot Operating System,"in Workshop on open source software International Conference of Robotic and Automation, Kobe Japan, 2009. 